\title{
DEVELOPMENT OF VIRTUAL PIPE SIMULATION SYSTEM FOR INSPECTION ROBOT DESIGN
}

\author{
Miura, Satoshi (1); Kawamura, Kazuya (2); Fujie, Masakatsu (1); Sugano, Shigeki (1); Miyashita, \\ Tomoyuki (1)
}

1: Waseda University; 2: Chiba University

\begin{abstract}
Pipe inspection robots have been developed to reduce the cost and time required for gas pipe inspection. However, these robots have been developed using a scrap and build method and are not used in practice. In this paper, we propose a method of virtual pipe inspection simulation to clarify the parameters that are important in increasing the robot's ease of use. This paper presents the results obtained by a feasibility study with regard to pipe simulation. We developed a virtual pipe by simulating eight actual turns of an external gas pipe, and a robot equipped with camera at the tip. In the experiments, three individuals working in the field of gas inspection carried out the operation. We obtained questionnaire, time, and brain activity data. The results revealed various important points that must be considered in practical simulation and robot design. In conclusion, the virtual pipe simulation can be useful in developing the design of a pipe inspection robot.
\end{abstract}

Keywords: Simulation, Design for interfaces, Human behaviour in design, Infrastructure

\author{
Contact: \\ Miura, Satoshi \\ Waseda University \\ Mechanical Engineering \\ Japan \\ miura.s@aoni.waseda.jp
}

Cite this article: Miura, S., Kawamura, K., Fujie, M., Sugano, S., Miyashita, T. (2019) 'Development of Virtual Pipe Simulation System for Inspection Robot Design', in Proceedings of the 22nd International Conference on Engineering Design (ICED19), Delft, The Netherlands, 5-8 August 2019. DOI:10.1017/dsi.2019.368 


\section{INTRODUCTION}

Pipelines support the gas supply to each house. In Japan, it is said that all gas pipelines buried underground extend for more than 1.5 billion km (Gas Pipe Overview, 2018). Generally, gas is sent through the main pipe under the public road and branches to an external gas pipe at a residential site. A 50A (diameter of $52.9 \mathrm{~mm}$ ) pipe is used as the main pipe, while a 25A (diameter of $27.6 \mathrm{~mm}$ ) pipe is used as the external gas pipe (Gas Pipe Overview, 2018), as shown in Figure 1. The external gas pipe includes four sets of two turns consisting of a bend and an elbow (Gas Pipe Overview, 2018). Because these gas pipes are buried underground, they are poorly maintained. Therefore, corrosion often appears inside the gas pipe as the pipe ages (Cole and Marney, 2012). The most common approach toward inspecting the interior of a gas pipe is as follows: the inspector stops the gas supply, digs up the pipe branching from the main road, inserts an endoscope into the pipe and inspects its interior (Ogai and Bhattacharya, 2018). The entire process is carried out in approximately 180 hours and requires that the gas supply is disrupted during the gas pipe inspection. Moreover, digging up and reburying the road entails costs including personal expenses and catering (Cole and Marney, 2012).

Various researchers have investigated a method of analysing the corrosion (Sinha et al., 2003; Iyer and Sinha, 2006; Najjaran et al., 2006; Schoefs et al., 2009; Tran et al., 2009; Duchesne et al., 2013; Cha et al., 2018). Moreover, such a method must be capable of monitoring the pipe's interior (Hsu and Mohan, 1991; Sinha and Pandey, 2002; Cheraghi et al., 2005; Dridi et al., 2008; Esposito et al., 2015). Many studies have been conducted on gas pipe inspection using three types of inspection methods, as shown in Figure 2. One such method is the pumping type. For example, recently, Okamura developed a soft robot using the gas pressure feeding type (Hawkes et al., 2017). Fukuda developed a gas pressure type robot using a hydrogen storage alloy (Fukuda and Engneering, 1989). Another method type is the pushing type. This is the simplest but most popular method. Olympus developed the flexible microscope IPLEX, which is inserted by the inspector into the pipe to observe whether corrosion exists (Videoscopes and Borescopes IPLEX RX/IPLEX RT, 2018). In fact, the IPLEX has been used in the field. Another type is the self-propelled method. Komori developed a wheel type inspection robot with Tokyo Gas (Komori and Suyama, 2012). Bertetto developed the inch-worm type flexible robot (Bertetto and Ruggiu, 2001). Thus, pipe inspection methods have been investigated extensively. However, none of the pipe inspection or robot development methods have been established because these mechanisms are not practical. In fact, these methods have been developed using the scrap and build method.

In this paper, the authors propose a method using a virtual pipe inspection simulation to gain insight into the design of a practical robot, as shown in Figure 3. Before designing a robot, while a user operated the virtual pipe inspection robot in the simulation, the authors measured the user's performance in terms of the duration of the operation, operation accuracy, and brain activity. The user changed the robot mechanisms and parameters to increase the ease of operating the robot. The authors evaluated and configured the important mechanical parameters. After validation had been carried out through a virtual reality (VR) simulation, the authors designed and constructed the robot and interface. The objective of this study was to assess the feasibility of developing a virtual pipe inspection simulation. Hence, the authors developed a virtual pipe and an inspection robot that simulated the actual pipe inspection process. In the experiments, the authors obtained user feedback, the duration of the operation, and brain activity data for three individuals working in the field of gas pipe inspection. Brain activity was measured to evaluate the ease of operation as perceived by the users. The brain activity data indicated the design factor required to increase the ease of operation. Thus, the authors clarified the important parameters in the design of a practical interface and robot.

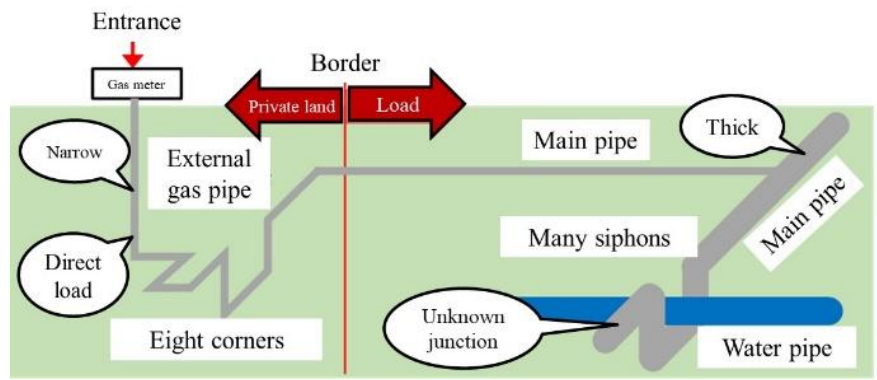

Figure 1. Gas pipeline 


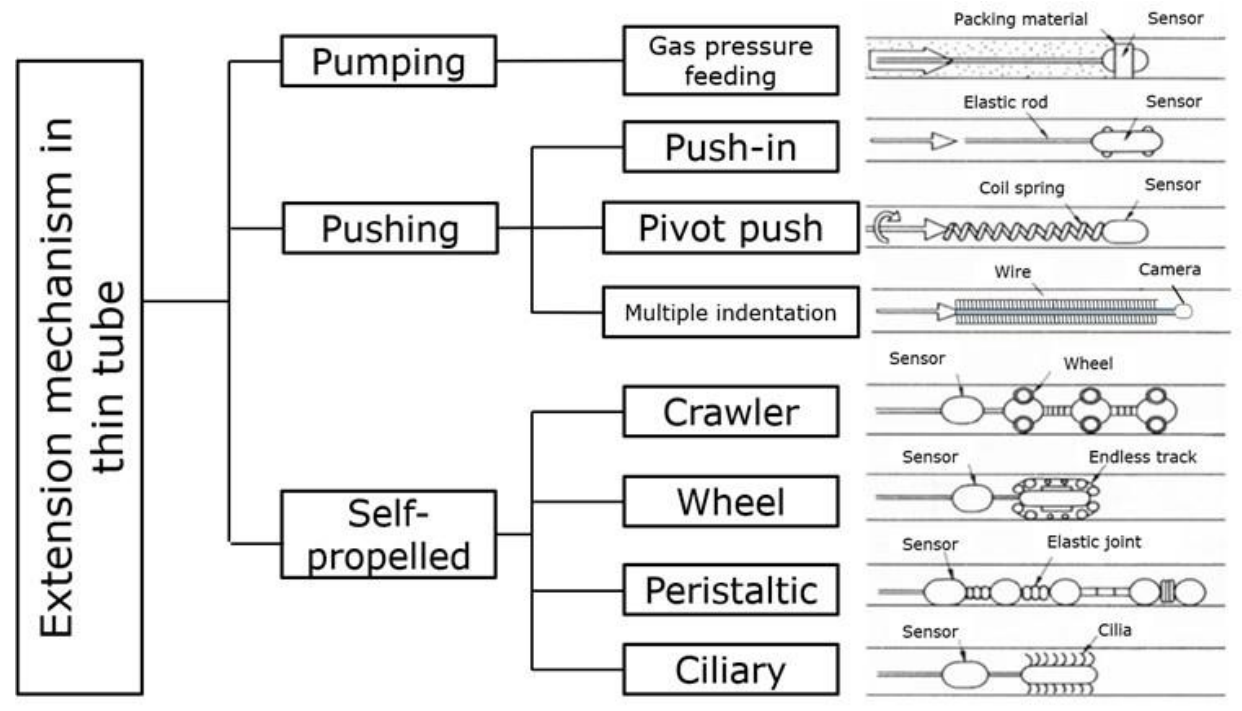

Figure 2. Conventional pipe robot variation

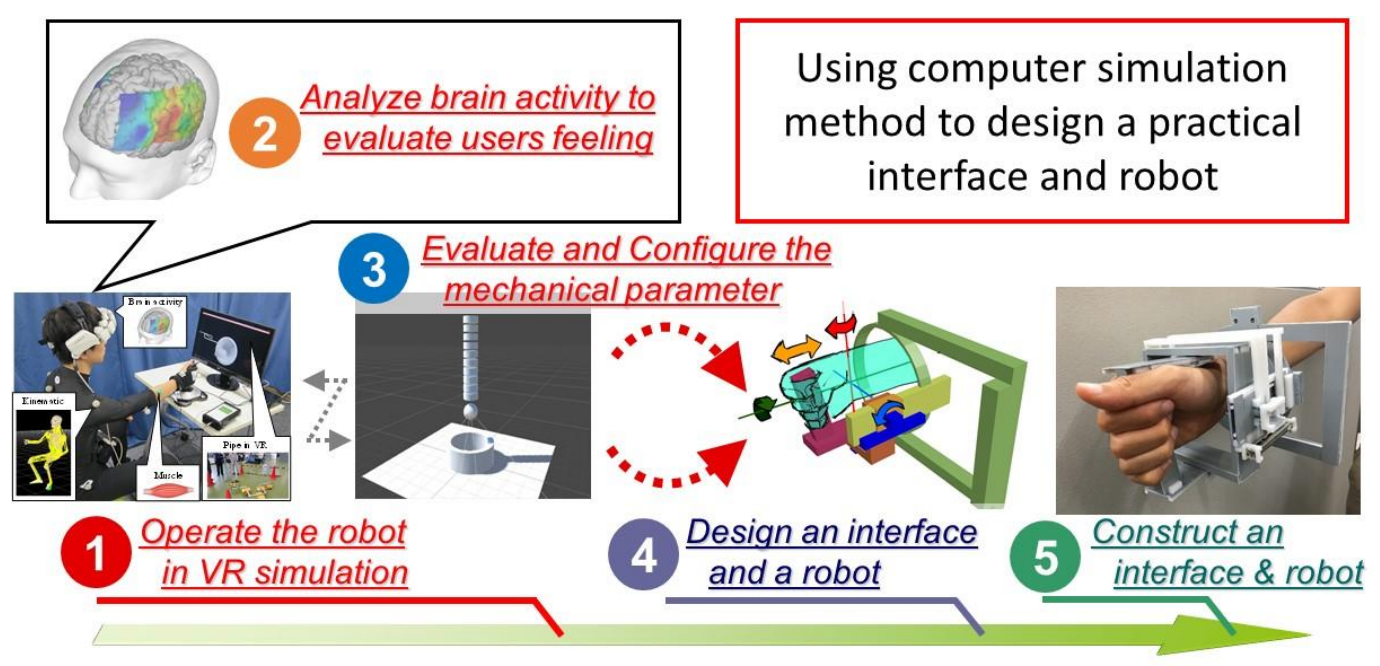

Figure 3. Proposed design method

\section{PIPE INSPECTION SIMULATION}

In this study, the authors developed a pipe inspection simulation. The target pipe was the external gas pipe shown in Figure 4. The external gas pipe consisted of one straight pipe and eight bent pipes, as shown in Figure 5. The simulation was developed using the three combinations shown in Figure 3. As can be seen, the first part was straight with two bent pipes. The second part comprised two bent pipes followed by another two bent pipes. The third part was straight with two bent pipes followed by another two bent pipes.

The simulation was developed using Unity (Unity technologies, USA) and C\#. The physics engine was PhysX (NVIDIA, USA). The robot consisted of a sphere and ten cylinders, and was controlled by the users to inspect the pipe interior, as shown in Figure 6. This robot simulated a pipe inspection robot equipped with a camera at the tip. The viewpoint was set at the tip of the sphere. The sphere and the cylinders were connected by a spring and damper. The diameters of the sphere and cylinder were $10.0 \mathrm{~mm}$ 

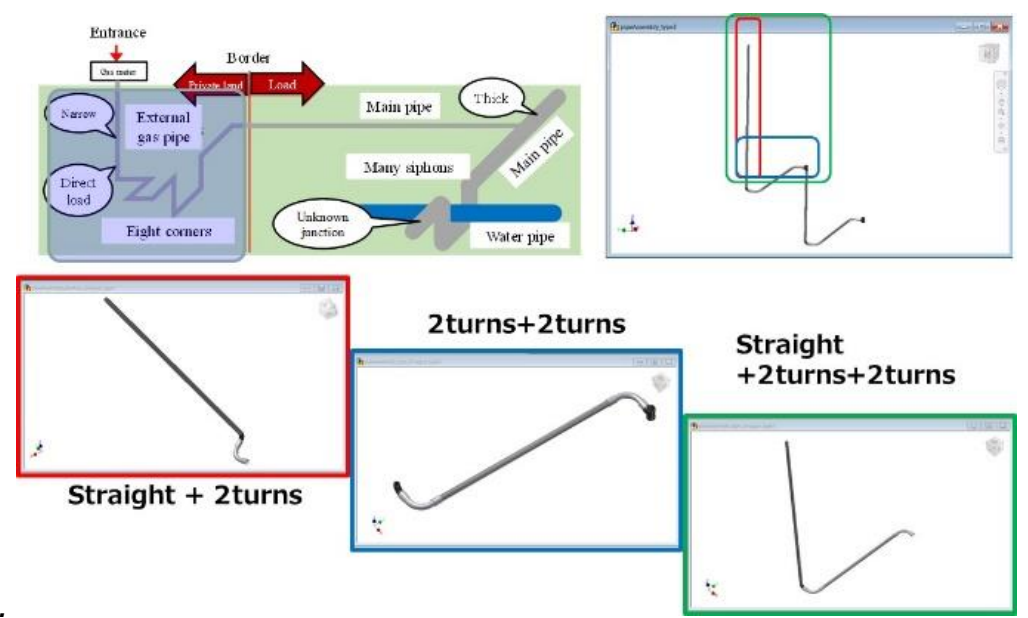

Figure 4. Target pipe

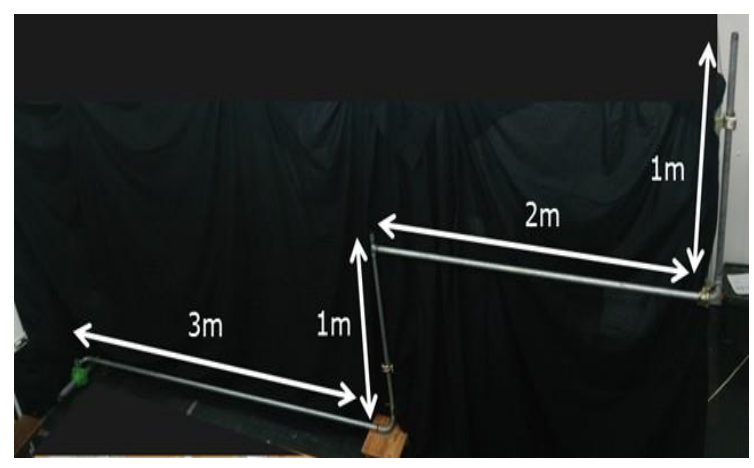

(a) Actual pipe

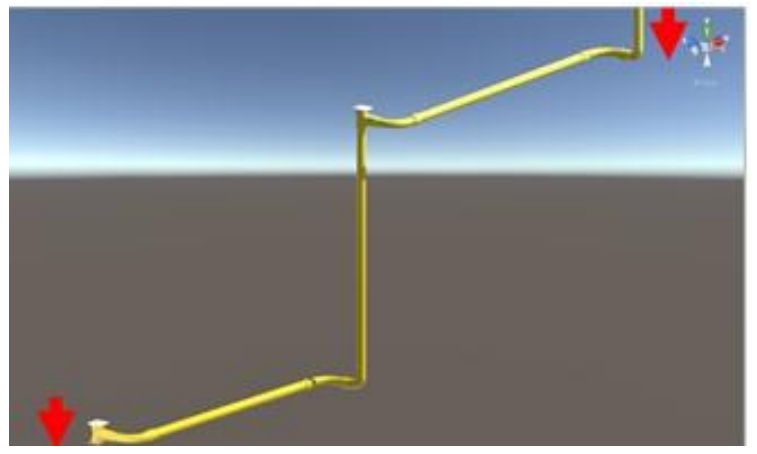

(b) Virtual pipe

Figure 5. External gas pipe specification

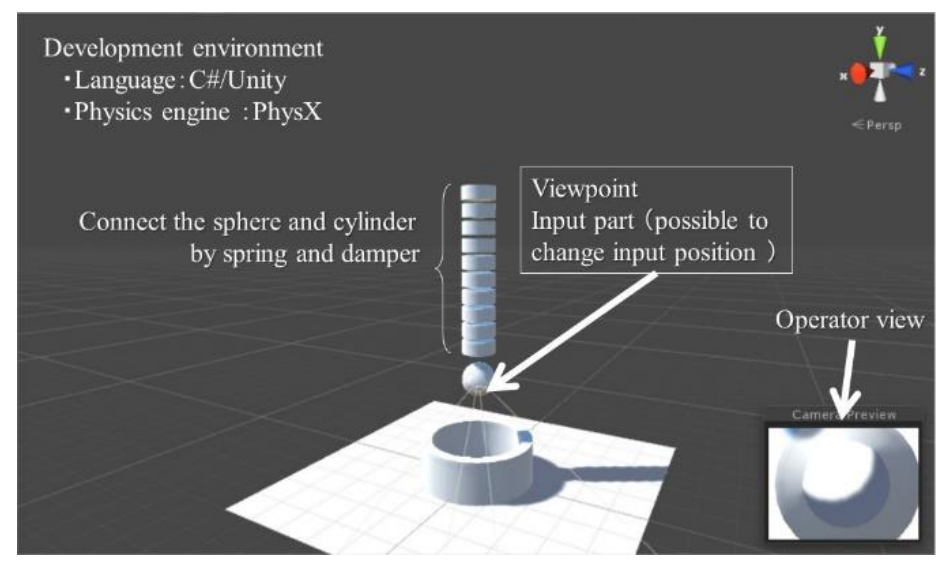

Figure 6. Virtual pipe simulation

The user controlled the sphere in the simulator using a joystick (Logitech Extreme 3D Pro). The sphere had four degrees of freedom: moving forward, rotating pitch, yaw, and roll, as shown in Figure 7. When the joystick was tilted, the sphere posture was input as the pitch and yaw rotation. As the joystick twisted, the roll rotation was input. The sphere advanced at a speed corresponding to the lever's inclination amount. The sphere could also move backwards. 


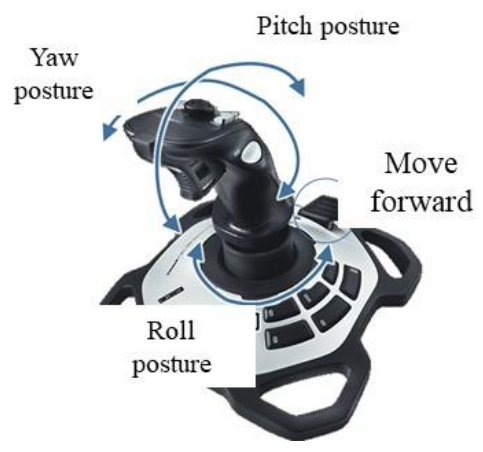

(a) Logitech Extreme 3D Pro

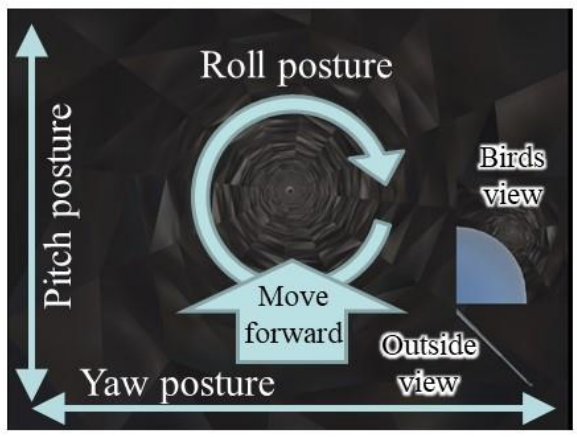

(b) Operators view

Figure 7. Simulation operation method

\section{EVALUATION METHOD}

To evaluate the user's perception with regard to the ease of operating the VR simulation, it was necessary to measure the user's perception with regard to the pipe interior. In the field of cognitive science, brain activity is useful in determining how a human feels, for the purposes of mental diagnosis. However, brain activity is also considered in design evaluation. For example, various studies have reported that brain activity has been measured to evaluate the quality of automobile rides (Harada et al., 2005). In master-slave operation, the user watches the slave robot on the screen; therefore, the user perceives the slave movement as visual information delivered through visual stimuli. Visual information was input to the occipital cortex. Moreover, the user moved a master console to perceive the master movement as somatic information sensed by the hands. Somatic information was input to the parietal cortex. Both the visual and somatic information were processed in the prefrontal area, and output to the body through the cerebellum and spine. Therefore, in the prefrontal area, it is important that the master-slave movements coincide as visual and somatic sensory feedback, as shown in Figure 8. The prefrontal cortex carries out the integration process of various types of information. Therefore, because the visual and somatic senses do not match, the processing of information becomes slow such that the prefrontal cortex is strongly activated.

The authors measured the prefrontal cortex using a wearable brain imaging device to show how intuitively the user perceived the coincidence of visual and somatic sensory feedback. The less the prefrontal cortex was activated, the greatest was the ease of operation perceived by the user.

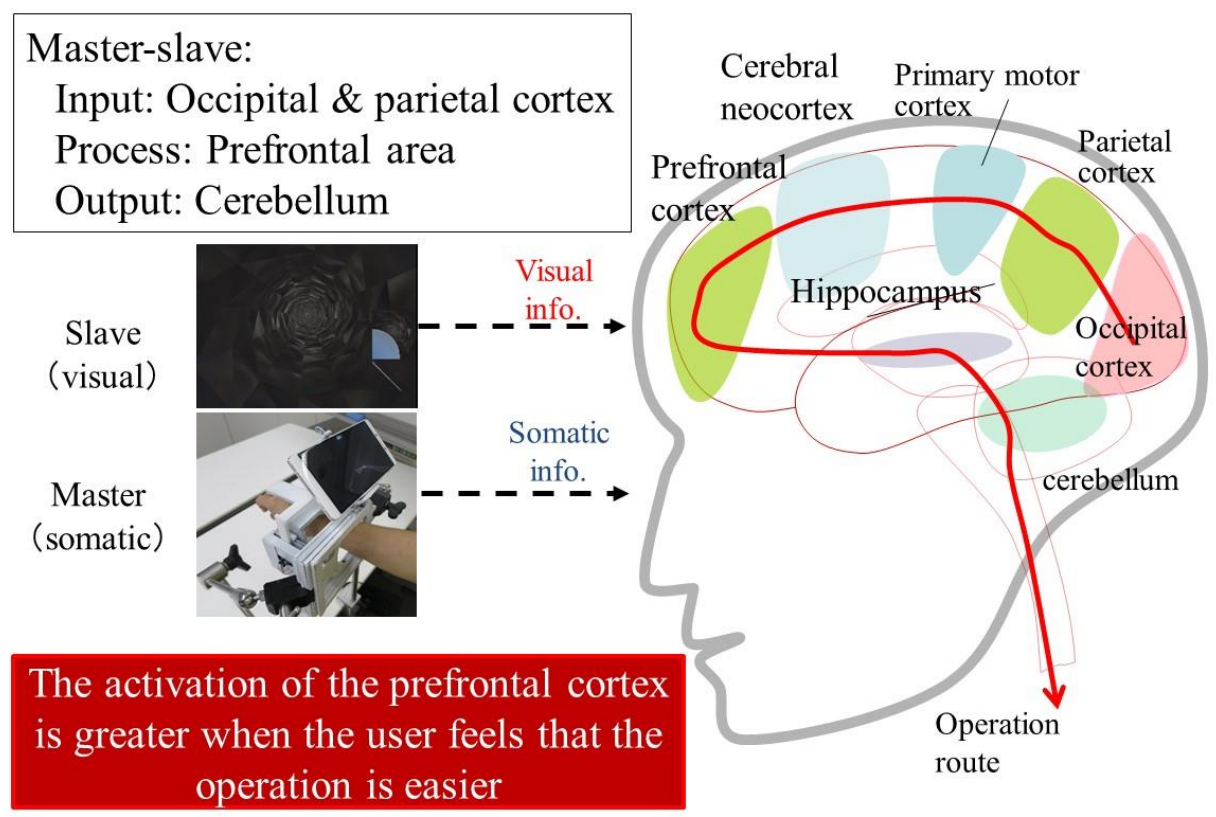

Figure 8. Method of evaluating brain activity 


\section{EXPERIMENT}

Three individuals participated in the experiment, as shown in Figure 9. The participants were staff members of Tokyo Gas, Co., Ltd. Two of the participants were experts, while the third participant was a novice in the field of gas pipe inspection. All experiments involving human subjects were performed in accordance with the 1964 Helsinki Declaration. The authors obtained informed consent from all participants.

The test course comprised four patterns. The first pattern was straight. The second pattern was straight with two bent pipes. The third pattern had a straight part, two bent pipes, another straight part, and two bent and two cheese pipes. The fourth pattern had eight turns, as shown in Figure 5.

The participants responded to a questionnaire, and the authors obtained the operation time and brain activity results. The brain activity was measured using wearable optical topography equipment (WOT220, Hitachi Medico Inc., Japan) to evaluate the extent to which the participants felt that the operation was easy. Wearable optical topography measurement has the advantages of higher space resolution in comparison with an electroencephalogram (EEG), no magnetic influence, and portability (Sutoko et al., 2016; Sutoa et al., 2004). The brain activity was indicated by the oxygenated hemoglobin (oxy$\mathrm{Hb})(\mathrm{mm})$. The higher the brain activity was, the higher was the amount of oxy-Hb. The authors measured the prefrontal area because its function is to indicate the stress entailed in using an object (Carrieri et al., 2016; Matsuzaka et al., 2012).

The experimental procedure is presented below. The participant followed the straight course three times. For the other courses, the participant carried out the experiment until the objective had been achieved three times.

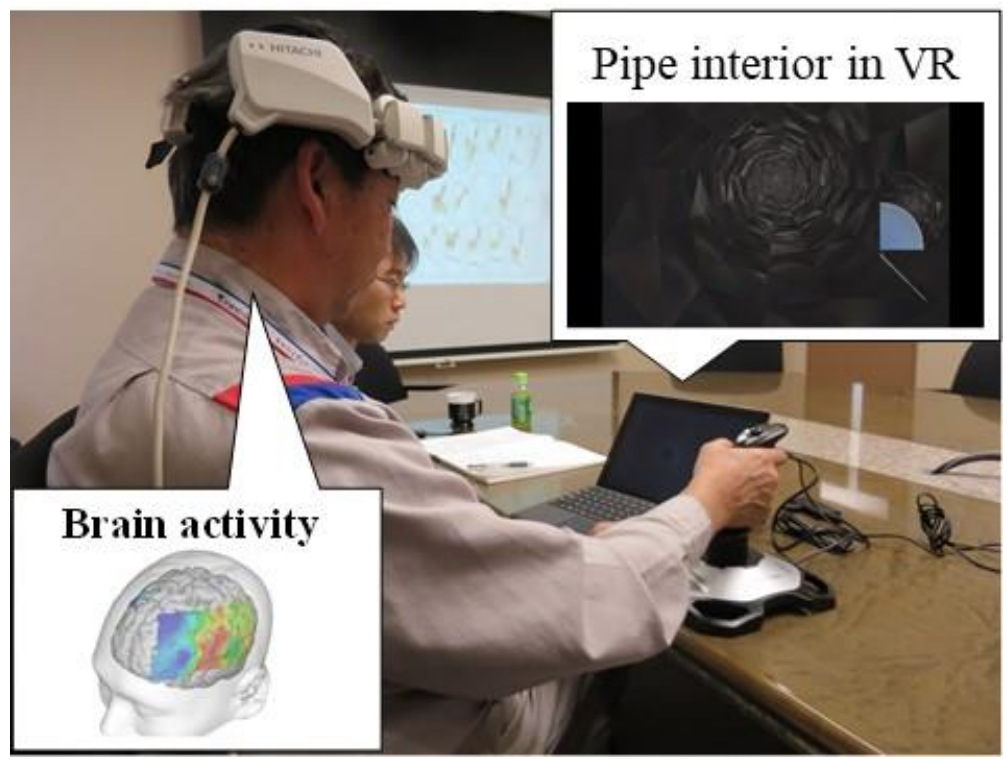

Figure 9. Overview of experiment

\section{RESULTS}

Figure 10 shows each question answered by the participants after the experiment with regard to the simulation. Figure 10(a-h) shows the answers given by the three participants to the following questions during the three stages.

(a) Can it conduct an actual pipe inspection?

(b) Do you feel as if you are watching the interior of an actual pipe?

(c) How close is the simulation to an actual pipe inspection?

(d) Are the movement viewpoints similar to those of an actual pipe inspection?

(e) How does the speed feel?

(f) How do you feel about the viewpoint rotation?

(g) Are you irritated by the unintentional rotation?

(h) Is the navigation necessary?

Figure 10(i) shows the order of the design factors by degree of importance according to the three participants' responses to the questionnaire: viewpoint angle, viewpoint position, console mechanism, 
viewpoint moving speed, friction by pipe, appearance inside a pipe, console configuration, navigation, and delay time. The higher the value is, the more the participant perceived the items to be important. Figure 11 shows the time duration for each pipe course. Sections 1-8 represent the time of passing through each section. Figure 12 shows the longitudinal oxy-Hb data for each straight and curve interval.

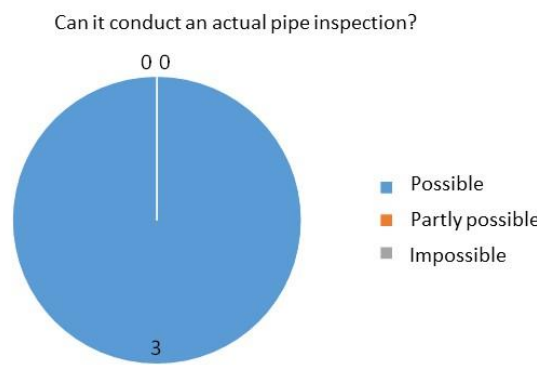

(a) Can it conduct an actual pipe inspection?

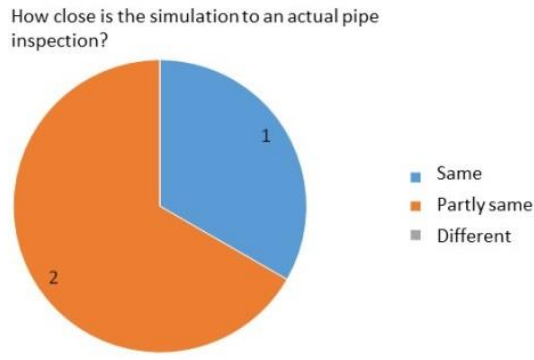

(c) How close is the simulation to an actual pipe inspection?

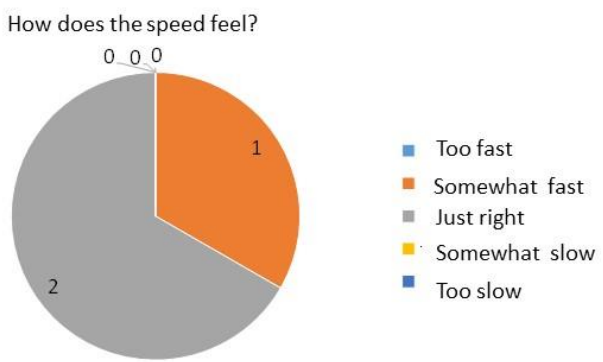

(e) How does the speed feel?

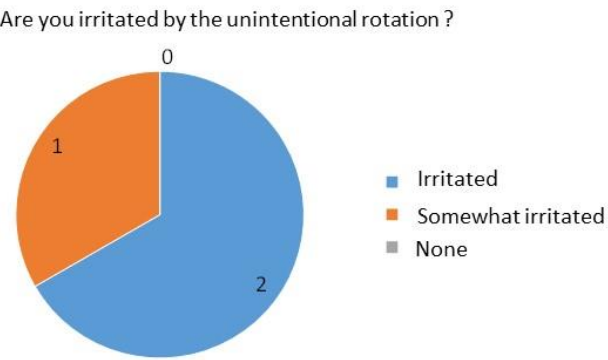

(g) Are you irritated by the unintentional rotation?

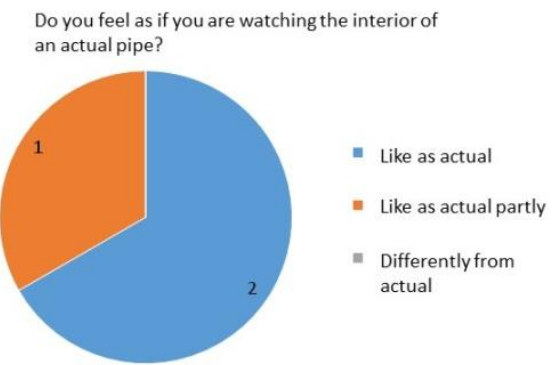

(b) Do you feel as if you are watching the interior of an actual pipe?

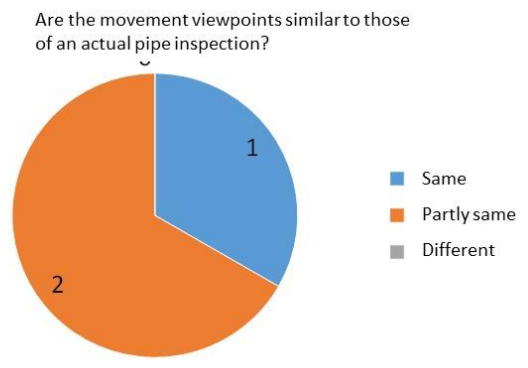

(d) Are the movement viewpoints similar to those of an actual pipe inspection?

How do you feel about the rotation of viewpoint?

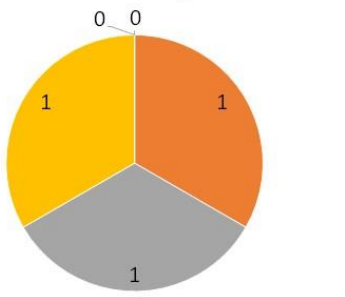

-10ofast

- Somewhat fast

Just right

Somewhat slow

- Too slow

(f) How do you feel about the viewpoint rotation?

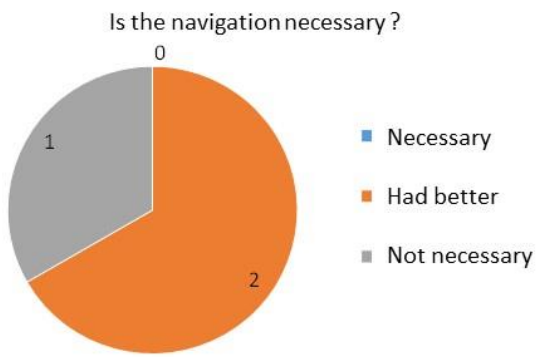

(h) Is the navigation necessary? 

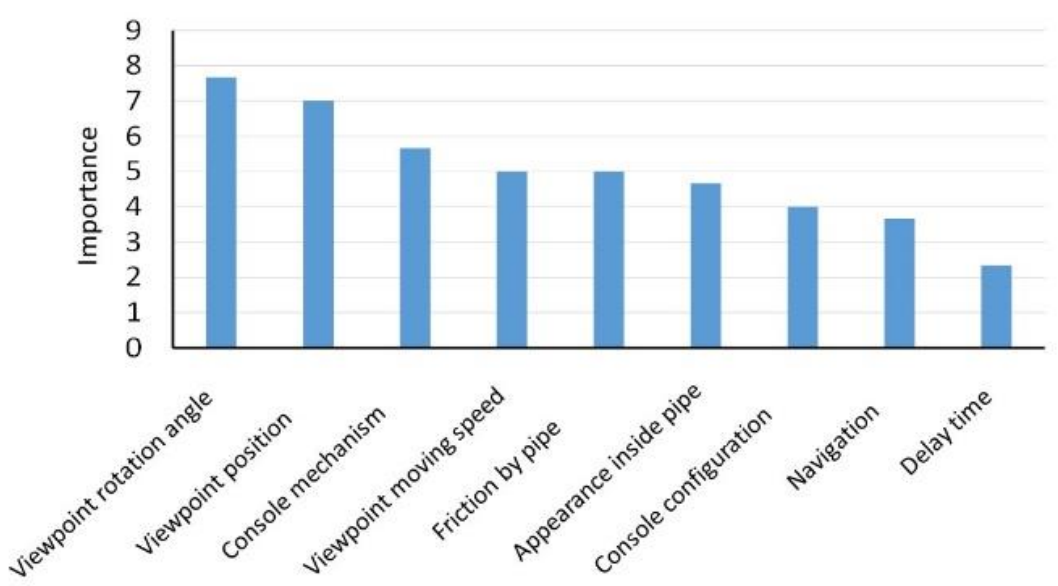

(i) Importance of simulator improvements

Figure 10. Questionnaire results

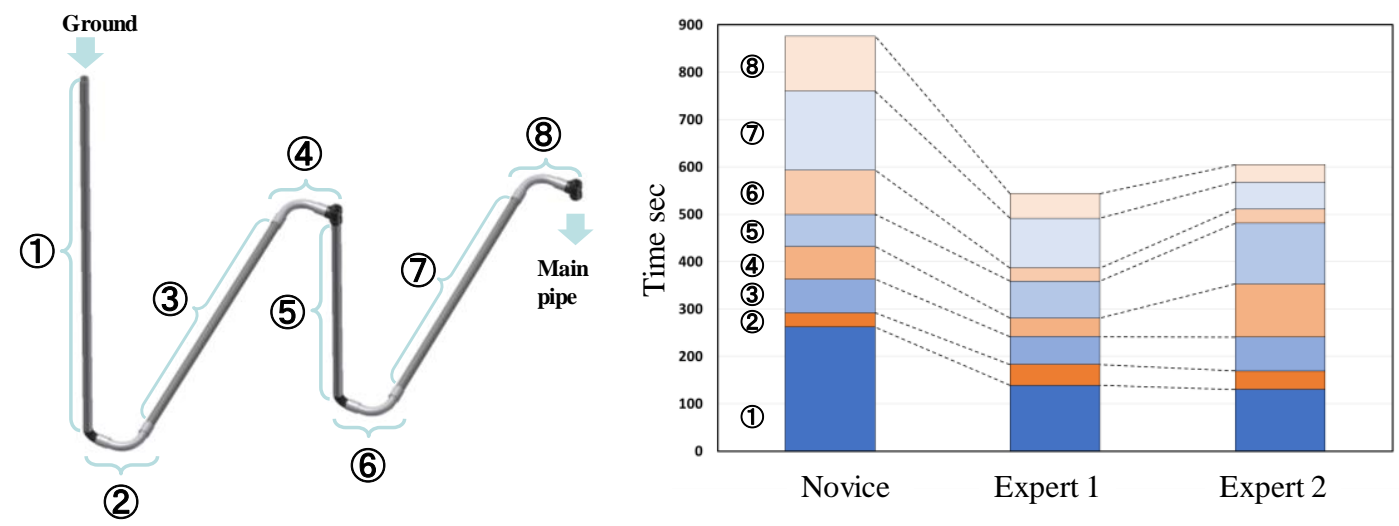

Figure 11. Time results

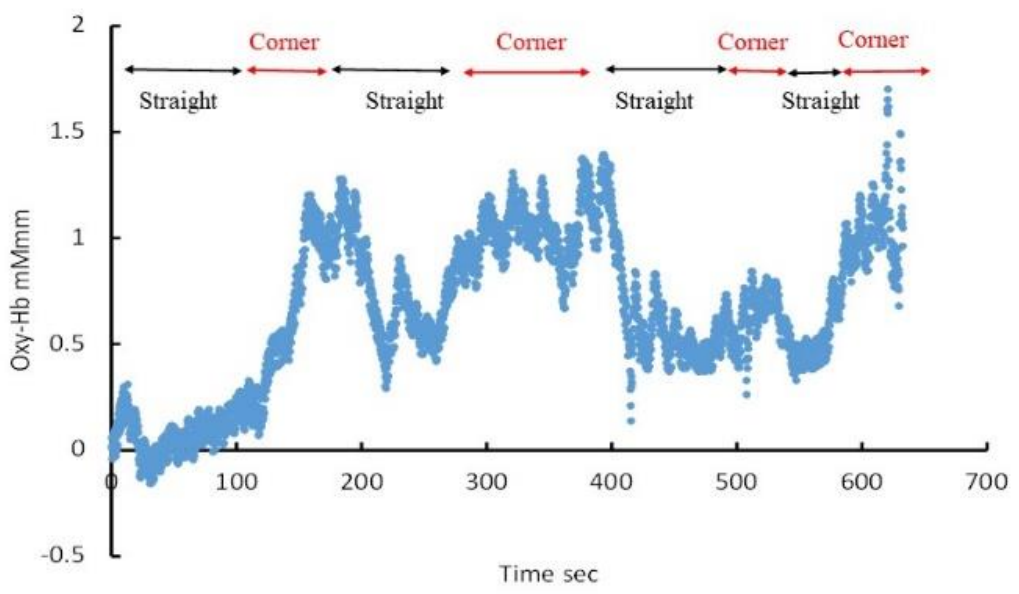

Figure 12. Brain activity at prefrontal area

\section{DIscussion}

Figure 10(a) and (b) shows the feasibility of the simulation. All participants responded that the simulation corresponded to an actual pipe inspection. Additionally, two of the three participants felt as if they were watching the interior of an actual pipe. The third participant felt partly similar to watching the interior of an actual pipe. 
Furthermore, Figure 10(c) and (d) shows the user satisfaction in relation to appearance. The simulation perceived to correspond to an actual simulation was given the value of one; the simulation perceived to partly correspond to an actual simulation was given the value of two. Similarly, the viewpoint movement perceived to be the same as the actual movement was given the value of one; the movement perceived to partly correspond to the actual movement was given the value of two.

Figure 10(e-h) shows the comments related to the operation. The forward and rotation speeds were different for each participant, as shown in Figure 10(e) and (f). This suggests that it is necessary to adjust the tuning of individual differences in the design of the pipe inspection robot. Furthermore, as the viewpoint went forward, the viewpoint rotated in the roll direction unintentionally, even though the participant did not input the roll rotation. The unintentional rotation was caused by the reaction force from the pipe. This irritated two of the participants, and somewhat irritated the third participant, as shown in Figure $10(\mathrm{~g})$. Therefore, the unintentional rotation should be improved. Moreover, it is thought that navigation such as mapping is not important for easy operation.

Figure 10(i) shows the degree of importance for the improvements required by the simulator, and indicates the importance of the pipe inspection robot. The viewpoint rotation angle was considered as the most important parameter by the participants. Moreover, the viewpoint position was considered as the second most important parameter. Thus, the console mechanism and viewpoint moving speed were considered as important. These parameters were related to the operation. Therefore, the operation was more important than the friction, appearance, and navigation, and this result should be considered in the robot's design.

From Figure 11, the duration of the operation by the experts was shorter than the duration of the operation by the novice. In particular, the durations were greatly different for the first straight part, because the novice participant exercised caution in operating the simulator. Moreover, all of the participants needed more time at the corners. In Figure 12, it can be seen that the oxy-Hb was higher at the corner in comparison with the straight part. The higher the oxy-Hb was, the greater was the stress experienced by the participant. Therefore, the participants experienced less stress at the straight part and more stress at the corner. Because two turns occurred continuously in one set, after one turn, the participant had to operate such that they could enter the second turn. This indicates that the robot design must be improved such that it is easier to bend around corners.

\section{CONCLUSION}

The authors propose a virtual pipe inspection simulation to gain insight into the design of an effective pipe inspection robot. In this study, the authors simulated an external gas pipe that consisted of eight turns, and a robot equipped with a camera at the tip. In the experiments, three participants working in the field of pipe inspection operated the simulator. Based on the obtained results, it was concluded that the practical application of the simulation is feasible. Additionally, the questionnaire, time, and brain activity results revealed the important items to consider in the robot design.

\section{REFERENCES}

Bertetto, A.M. and Ruggiu, M. (2001), "In-pipe inch-womn pneumatic flexible robot", IEEVASME International Conference on Advanced Intelligent Mechatronics Proceedings, pp. 1226-1231.

Carrieri, M., et al. (2016), "Prefrontal Cortex Activation Upon a Demanding Virtual Hand-Controlled Task: A New Frontier for Neuroergonomics", Frontiers in Human Neuroscience, Vol. 10 No. February, pp. 1-13. https://doi.org/10.3389/fnhum.2016.00053.

Cha, Y.J., et al. (2018), “Autonomous Structural Visual Inspection Using Region-Based Deep Learning for Detecting Multiple Damage Types”, Computer-Aided Civil and Infrastructure Engineering, Vol. 33 No. 9 , pp. 731-747. https://doi.org/10.1111/mice.12334.

Cheraghi, N., Zou, G.P. and Taheri, F. (2005), "Piezoelectric-based degradation assessment of a pipe using fourier and wavelet analyses", Computer-Aided Civil and Infrastructure Engineering, Vol. 20 No. 5, pp. 369-382. https://doi.org/10.1111/j.1467-8667.2005.00403.x.

Cole, I.S. and Marney, D. (2012), The science of pipe corrosion: A review of the literature on the corrosion of ferrous metals in soils, Corrosion Science. Elsevier Ltd, Vol. 56, pp. 5-16. https://doi.org/10.1016/j.corsci.2011.12.001.

Dridi, L., et al. (2008), "Using evolutionary optimization techniques for scheduling water pipe renewal considering a short planning horizon", Computer-Aided Civil and Infrastructure Engineering, Vol. 23 No. 8, pp. 625-635. https://doi.org/10.1111/j.1467-8667.2008.00564.x. 
Duchesne, S., et al. (2013), "A Survival Analysis Model for Sewer Pipe Structural Deterioration”, ComputerAided Civil and Infrastructure Engineering, Vol. 28 No. 2, pp. 146-160. https://doi.org/10.1111/j.14678667.2012.00773.x.

Esposito, S., et al. (2015), "Simulation-Based Seismic Risk Assessment of Gas Distribution Networks", Computer-Aided Civil and Infrastructure Engineering, Vol. 30 No. 7, pp. 508-523. https://doi.org/10.1111/mice.12105.

Fukuda, T. and Engneering, M. (1989), "Rubber Gas Actuator Driven by Hydrogen Storage Alloy for In-pipe Inspection Mobile Robot with Flexible Structure", Proceedings of the IEEE International Conference on Robotics and Automation (ICRA), pp. 1-6.

Gas Pipe Overview (2018), Tokyo Gas, Available at: https://home.tokyo-gas.co.jp/gas/koji/zentaizou.html

Hawkes, E.W., et al. (2017), “A soft robot that navigates its environment through growth", Science Robotics, Vol. 2 No. 8, pp. 1-8. https://doi.org/10.1126/scirobotics.aan3028.

Hsu, L. and Mohan, S. (1991), "Computer Aided Configuration of Pipe Systems in Buildings", Computer-Aided Civil and Infrastructure Engineering, Vol. 6 No. 1, pp. 15-26. https://doi.org/10.1111/j.14678667.1991.tb00394.x.

Iyer, S. and Sinha, S.K. (2006), "Segmentation of pipe images for crack detection in buried sewers", ComputerAided Civil and Infrastructure Engineering, Vol. 21 No. 6, pp. 395-410. https://doi.org/10.1111/j.14678667.2006.00445.x.

Komori, M. and Suyama, K. (2012), "Inspection robots for gas pipelines of Tokyo Gas", Advanced Robotics, Vol. 1864 No. 2001, https://doi.org/10.1163/156855301300235922.

Matsuzaka, Y., et al. (2012), "Neuronal activity in the primate dorsomedial prefrontal cortex contributes to strategic selection of response tactics", Proceedings of the National Academy of Sciences, Vol. 109 No. 12, pp. 4633-4638. https://doi.org/10.1073/pnas.1119971109.

Najjaran, H., Sadiq, R. and Rajani, B. (2006), "Fuzzy expert system to assess corrosion of cast/ductile iron pipes from backfill properties", Computer-Aided Civil and Infrastructure Engineering, Vol. 21 No. 1, pp. 67-77. https://doi.org/10.1111/j.1467-8667.2005.00417.x.

Ogai, H. and Bhattacharya, B. (2018), Pipe Inspection Robots for Structural Health and Condition Monitoring, Springer.

Schoefs, F., et al. (2009), "Comparison of additional costs for several replacement strategies of randomly ageing reinforced concrete pipes”, Computer-Aided Civil and Infrastructure Engineering, Vol. 24 No. 7, pp. 492508. https://doi.org/10.1111/j.1467-8667.2009.00603.x.

Sinha, S.K., Fieguth, P.W. and Polak, M.A. (2003), "Computer vision techniques for automatic structural assessment of underground pipes”, Computer-Aided Civil and Infrastructure Engineering, Vol. 18 No. 2, pp. 95-112. https://doi.org/10.1111/1467-8667.00302.

Sinha, S.K. and Pandey, M.D. (2002), "Probabilistic neural network for reliability assessment of oil and gas pipelines", Computer-Aided Civil and Infrastructure Engineering, Vol. 17 No. 5, pp. 320-329. https://doi.org/10.1111/1467-8667.00279.

Sutoa, T., et al. (2004), "Mulitchannel Near-infrared Spectroscopy in Depression and Schizophrenia", Biological Psychiatry, Vol. 55 No. 1, pp. 501-511.

Sutoko, S., et al. (2016), "Tutorial on platform for optical topography analysis tools", Neurophotonics, Vol. 3 No. 1, pp. 010801. https://doi.org/10.1117/1.NPh.3.1.010801.

Tran, D.H., Perera, B.J.C. and Ng, A.W.M. (2009), "Comparison of structural deterioration models for stormwater drainage pipes", Computer-Aided Civil and Infrastructure Engineering, Vol. 24 No. 2, pp. 145-156. https://doi.org/10.1111/j.1467-8667.2008.00577.x.

Videoscopes and Borescopes IPLEX RX/IPLEX RT (2018), Olympus, Available at: https://www.olympusims.com/en/rvi-products/iplex-rx/.

\section{ACKNOWLEDGMENTS}

This work was supported in part by the Council for Science, Technology, and Innovation (CSTII) Cross-ministerial Strategic Innovation Promotion Program (SIP) (Funding agency: JST). The authors appreciate the assistance of Tokyo Gas Co, Ltd. The authors thank Edanz Group (www.edanzediting.com/ac) for editing a draft of this manuscript. 